\title{
Grupo técnico de informação em saúde e populações: contribuições para a política nacional de informação e informática em saúde ${ }^{1}$
}

\section{Ricardo Bezerra Cavalcante}

Bolsista de Pos-doutorado júnior (CNPQ) em Ciência da Informação - ECI/UFMG. Doutorado em Ciência da Informação - ECI/UFMG. Professor Adjunto II da Universidade Federal de São João Del Rei - Campus Centro Oeste Dona Lindu.

Marta Macedo Kerr Pinheiro

Doutorado em Ciência da Informação IBICT/CNPq-ECO-UFRJ e doutorado em Sociologie - Centre dÉtudes des Mouvements Sociaux. Pósdoutorado em Ciência da Informação e da Comunicação pela Universidade Paul Sabatier/IUT/Toulouse III. Professor permanente da Universidade FUMEC e Professor colaborador do Programa de pós-Graduação em Ciência da Informação da Universidade Federal de Minas Gerais.

Yoshimi José Ávila Watanabe

Mestrando em Biotecnologia do Programa de Pósgraduação em Biotecnologia da Universidade Federal de São João Del Rei (PPGBiotec-UFSJ). Médico nefrologista e Cientista da Computação.

Cristiano José da Silva

Enfermeiro especialista em Informática em Saúde. Membro do Núcleo de Estudos e Pesquisas em Avaliação e Gestão em Saúde da Universidade Federal de São João Del Rei.

http://dx.doi.org/10.1590/1981-5344/1905

Estudo de abordagem qualitativa que teve como objetivo identificar e analisar as contribuições do Grupo Técnico de Informação em Saúde e Populações (GTISP) para a construção da Política Nacional de Informação e Informática em Saúde (PNIIS). Desenvolveu-se uma

\footnotetext{
${ }^{1}$ Artigo resultante da pesquisa de Pós-Doutorado "A política nacional de informação e informática em saúde nas esferas federal, estadual e municipal de gestão do Sistema Único de Saúde". Pesquisa financiada pelo Conselho Nacional de Desenvolvimento Científico e Tecnológico (CNPQ).
} 
pesquisa documental a partir dos relatórios das oficinas de trabalho do GTISP, no âmbito dos Congressos Brasileiros de Saúde Coletiva, no período de 1992 a 2013. Após a análise preliminar, procedeu-se à Análise de Conteúdo dos documentos. Verificou-se que o GTISP tem contribuído para a construção da PNIIS propondo: a valorização do entono sócio-cultural que envolve a política de informação; a necessidade de construir uma política de informação para a área de forma coletiva, plural, pactuada e gerida de forma colegiada; o resgate da informação como um direito de todos e a necessidade de romper com o modelo de gestão da informação hegemônico no Brasil; a definição de planos diretores com vistas à suplantar as dificuldades reconhecidas no campo da informação e informática em saúde. Conclui-se que apesar das contribuições do GTISP para a construção da política de informação em saúde, a mesma ainda não foi legitimada. É preciso avançar em sua definição e reconhecimento em todas as esferas de governo, bem como no locus assistencial.

Palavras-chave: Política de informação; Informática em Saúde; Informação em Saúde

\section{Group technical information on health and population: contributions to the national information policy and health informatics}

This qualitative study aimed to identify and analyze the contributions of the Technical Group on Health Information and Populations for the construction of the National Information and Health Informatics. Developed a research document from the reports of the workshops of group under the Brazilian Congress on Collective Health, in the period 1992-2013. After preliminary analysis, we proceeded to the Content Analysis of the documents. It was found that the group has contributed to the construction of PNIIS proposing: the appreciation of the socio-cultural context involving information policy, the need to build an information policy for the area collectively, plural and collective management; redemption of information as a right for all and the need to break with the model of information management 
hegemonic in Brazil, the definition of master plans with a view to overcome the difficulties recognized in the field of information and health informatics. It is concluded that despite the contributions of group for building policy for health information, it has not been legitimized. You need to advance in their definition and recognition in all spheres of government as well as the locus of care.

\section{Keywords: Information Policy; Health Informatics;} Health Information.

Recebido em 20.02.2014 Aceito em 22.01.2015

\section{Introdução}

No ensejo de apoiar o desenvolvimento do campo da informação e informática em saúde no Brasil, o Grupo Técnico de Informação em Saúde e População (GTISP) da Associação Brasileira de Saúde Coletiva (ABRASCO), vem se constituindo num espaço de debates e proposições. Este grupo, dentre membros e convidados, é composto por pesquisadores, docentes, gestores, profissionais e outros representantes da sociedade, que se reúnem no âmbito das oficinas de trabalho dos congressos da ABRASCO e outros eventos. O grupo surgiu no âmbito do II Congresso Brasileiro de Epidemiologia realizado no ano de 1992, em Belo Horizonte, a partir da oficina de trabalho intitulada "Utilização de grandes bancos de dados nacionais" (ABRASCO; GTISP, 1994).

Espera-se que os documentos elaborados e publicizados pelo GTISP, ao longo de sua história, possam apontar elementos incorporados e a serem incorporados à Política Nacional de Informação e Informática em Saúde (PNIIS), bem como pode apresentar o contexto histórico e sociocultural que envolve o desenvolvimento desta política. Para Moraes e Vansconcellos (2005) o GTISP no decorrer de sua história vem destacando a necessidade de responder ao questionamento de como estabelecer para o país uma Política de informação. O grupo tem buscado então estabelecer um diálogo plural acerca desta política de maneira que se constitua em uma formulação coletiva.

Sabe-se que o sucesso de uma política de informação depende de uma elaboração compartilhada com vários setores da sociedade a fim de que seja reconhecida, legitimada e operacionalizada (KERR PINHEIRO, 2008). As políticas de informação nacionais tem o potencial de apontar caminhos que coloquem a informação a serviço das transformações ocorridas na sociedade (MARQUES; KERR PINHEIRO, 2011). Os autores ainda declaram que os estudos das políticas de informação podem auxiliar na apreensão de um contexto marcado pela confluência de fatores sociais, econômicos e tecnológicos.

Ressalta-se que, apesar dos 20 anos de SUS, ainda há que se avançar principalmente no caráter participativo da sociedade durante a 
definição de políticas públicas de saúde. Espera-se que a consolidação da PNIIS em todo território nacional possa contribuir para avanços esperados na operacionalização do Sistema Unico de Saúde (SUS).

No exercício da função gestora do Estado, a PNIIS, para ser a expressão da nação brasileira - uma república federativa - e em decorrência disso receber o nome de nacional, precisa ser estabelecida de fato, de forma articulada e solidária nas diversas instâncias sociais. Essas instâncias, fundamentais na construção do SUS, devem estar envolvidas num processo permanente de formulação, implementação e avaliação da política de informação que se deseja implantar. Neste sentido espera-se que a maturação do GTISP e sua evolução no que tange ao campo da Informação Informática em Saúde possam elucidar a própria evolução da PNIIS, bem como apontem contribuições para esta mesma política.

A partir do contexto elucidado, surge um questionamento: "Como o GTISP tem contribuído para a construção da PNIIS?" Assim, neste estudo, o objetivo foi de identificar e analisar as contribuições do GTISP para a construção da Política Nacional de Informação e Informática em Saúde.

\section{Métodos}

Estudo de abordagem qualitativa utilizando-se a pesquisa documental que se caracteriza pela "busca de informações em documentos [...] como relatórios, reportagens de jornais, revistas, cartas, filmes, gravações, fotografias, entre outras matérias de divulgação" (OLIVEIRA, 2007, p. 69). Para a autora, o documento é a fonte primária de dados a ser analisada, a partir do qual se tem uma relação direta com os fatos a serem analisados. Assim, "tudo o que é vestígio do passado, tudo o que serve de testemunho, é considerado como documento ou fonte" (CELLARD, 2008, p. 296). E mais: "pode tratar-se de texto escritos, mas também de documentos de natureza iconográfica e cinematográfica, ou de qualquer outro tipo de testemunho registrado, objetos do cotidiano, elementos folclóricos, etc." (CELLARD, 2008, p. 297). Também se pode qualificar de documento um relatório de entrevista, ou anotações feitas durante uma observação (CELLARD, 2008). Por fim, Appolinário (2009, p. 67), ainda define documento como sendo "Qualquer suporte que contenha informação registrada, formando uma unidade, que possa servir para consulta, estudo ou prova".

A justificativa para a escolha da pesquisa documental é a necessidade de captar a partir do percurso histórico do GTISP elementos que possam ter contribuído para a construção da política de informação em saúde. Para Cellard (2008) os documentos podem apresentar riquezas de informações que ampliam o entendimento de objetos cuja compreensão necessita de contextualização histórica e sociocultural. Outra justificativa para o uso da pesquisa documental é o fato de favorecer a observação do processo de maturação ou de evolução de indivíduos, grupos, conceitos, conhecimentos, comportamentos, mentalidades, práticas, entre outros (CELLARD, 2008). 
A escolha do GTISP deve-se ao fato de "constituir-se em um espaço aberto e plural de debate, construção e sistematização de propostas relacionadas a um Projeto Nacional para o campo da Informação em Saúde comprometido com a melhoria da Saúde da população brasileira" (ABRASCO; GTISP, 2008 p. 2). O grupo, por meio de suas análises e proposições apontou em 1993 a necessidade da pactuação de uma Política Nacional de Informação em Saúde (ABRASCO; GTISP, 1993). Além disso, também propôs alguns marcos referenciais para esta política. Os atores que compõem o GTISP são representantes de várias instâncias da sociedade: pesquisadores, docentes, bem como representantes do ministério da saúde. Além dos membros efetivos, as reuniões do grupo contam com a participação de convidados que representam os mais diversos setores da sociedade. Assim, os discursos que emanam deste grupo são fundamentais para constituição do corpus de análise neste estudo, pois pode configurar um debate diversificado, com representação de várias instâncias sociais e com percepções acerca do objeto PNIIS.

Os documentos que compuseram o corpus da pesquisa foram os Relatórios finais das oficinas de trabalho do GTISP realizadas no âmbito dos Congressos Brasileiros de Saúde Coletiva no período de 1992 a 2013. Espera-se que estes documentos apresentem as intenções acerca da Política de informação em desenvolvimento, bem como aponte os principais desafios inerentes a este processo de construção.

Em relação à organização e análise dos dados, procedeu-se primeiramente a análise preliminar dos documentos. Para Cellard (2008) há cinco dimensões que compõem a primeira etapa (análise preliminar) da análise documental. São eles: a avaliação do contexto em que foi produzido o documento; identificação dos autores, seus interesses e os motivos para a escrita do documento; avaliação da autenticidade e da confiabilidade do texto; avaliação da natureza do texto; identificação dos conceitos-chave e a lógica interna do texto. Todas estas cinco dimensões nortearam a primeira etapa da análise dos documentos selecionados.

Após a análise preliminar, procedeu-se a Análise de Conteúdo dos documentos que segundo Appolinário (2009) são técnicas de análise de dados linguísticos que se propõem em identificar os elementos fundamentais da comunicação, sendo enumerados e categorizados. Para Cellard (2008) a Análise de Conteúdo é muito utilizada na pesquisa documental. Este estudo obedeceu a resolução 196/96 do Conselho Nacional de Saúde e foi aprovado por comitê de ética em pesquisa segundo Parecer n0175.119/2012.

\section{Resultados}

\subsection{O GTISP na década de 1990: primeiras contribuições para a construção da PNIIS}

Em 1992 durante o II Congresso Brasileiro de Epidemiologia realizado em Belo Horizonte ocorreu a oficina de trabalho intitulada 
"Utilização de grandes bancos de dados nacionais" (ABRASCO; GTISP, 1994). O objetivo foi proporcionar reflexões sobre os usos dos bancos de dados nacionais envolvendo os desenvolvedores, gestores, profissionais de saúde e pesquisadores. Durante os debates identificaram-se várias dificuldades e desafios relacionados aos bancos de dados nacionais: variedade de bancos de dados e sistemas de informação desintegrados e desarticulados; dados estocados, sem produção de informações de qualidade; sistemas de informação sem documentação; insuficiência de recursos humanos e infraestrutura; centralização de informações no estado justificada pela necessidade do sigilo institucional.

Como resultado do esforço intelectual empreendido pelos componentes da oficina, produziram-se algumas recomendações. Para monitorar o cumprimento destas recomendações foi estipulada a necessidade de criação de um Comitê Técnico de Informações em Saúde junto à ABRASCO. Para este comitê foram definidas duas atribuições: elaborar propostas de padronização dos principais bancos de dados nacionais; elaborar um código de ética do uso da informação de interesse para a saúde. É neste momento que nasce o GTISP, já com grandes responsabilidades e um amadurecimento importante para as reflexões dos anos 1990. Em 1993, como resultado das reflexões, o GTISP desenvolveu o documento "Informação em saúde a serviço da sociedade" que contribuiu significativamente para 0 adensamento das discussões relacionadas ao campo da informação em saúde (ABRASCO; GTISP, 1993). Neste documento estão as primeiras citações explícitas acerca da política de informação em saúde que se desenvolveria ao longo dos anos.

Destaca-se que o GTISP enfatiza a necessidade da construção de uma política de informação de forma coletiva, democrática e gerida de forma colegiada. Também é proposto que a informação e a tecnologia não sejam tratadas nesta política de forma separada.

Faz-se necessário um novo pacto em torno de uma Política de Informação em Saúde, definida e coordenada por uma instância colegiada e democrática e operacionalizada por uma instância executora, que articule os vários agentes do complexo processo que envolve as atuais informações sóciodemográficas e de saúde (ABRASO; GTISP, 1993 p. 30).

Nesse sentido, a definição de uma Política de Informática em Saúde deve estar subordinada à Política de Informações em Saúde, não sendo possível separá-las (ABRASO; GTISP, 1993, p. 34).

É nesta direção que a proposição por uma nova Política de Informações em Saúde (PIS) se coloca articulada a uma ampla Política de Informações. Não se trata, simplesmente, de alcançar, como marco inicial, a assinatura de uma Portaria 
Ministerial ou uma Resolução, a ser publicada no Diário Oficial. Mas, primordialmente, a definição de uma estratégia delineada para o PROCESSO de construção de uma Política de Informações no Brasil, a partir de "negociações" em busca de entendimentos progressivos entre os principais interlocutores envolvidos, representantes da sociedade organizada e do governo [...] (ABRASO; GTISP, 1993 p. 35).

No V Congresso Brasileiro de Saúde Coletiva, em Águas de Lindóia, em 1997, o GTISP promoveu a oficina de trabalho "Compatibilização de Bases de Dados Nacionais" (ABRASCO; GTISP, 1997). Nesta oficina o objetivo foi de elaborar propostas para a compatibilização dos sistemas de informação de abrangência nacional. Os seguintes pontos foram tratados: Preservação das bases de dados nacionais no processo de descentralização; Definição das variáveis necessárias para cada nível de gestão; Necessidade de mudanças nas estruturas de informações e nos cadastros de estabelecimentos de saúde; Discussão sobre os processos éticos em todo o fluxo informacional; Estudos de compatibilização do número único de identificação do indivíduo, instituído com o cartão SUS.

Como resultado desta oficina de trabalho foi proposta a criação de uma Comissão Nacional de Informações em Saúde no âmbito do Conselho Nacional de Saúde, e que fosse responsável por propor questões relacionadas à constituição de uma política nacional de informação em saúde. Ainda, como resultado desta oficina do GTISP, foi criado o Comitê Temático Interdisciplinar (CTI) de Compatibilização dos sistemas e bases de dados nacionais que elaborou os parâmetros necessários para a padronização dos sistemas de informação em saúde. Em 1998, por meio da Portaria 3.947/GM de 25 de Novembro de 1988 e corrigida em 14 de janeiro de 1999, grande parte desses parâmetros elaborados pelo comitê foram reconhecidos (BRASIL, 1999).

\subsection{O GTISP no ano 2000: por um processo coletivo, participativo e democrático na formulação da PNIIS.}

Em 2000 o GTISP promoveu no âmbito do VI Congresso Brasileiro de Saúde Coletiva a Oficina de Trabalho "Informações para a Gestão do SUS: necessidades e perspectivas" (ABRASCO; GTISP, 2000). A busca por uma política de informação em saúde continuou, como nos anos anteriores, sendo um dos objetivos do GTISP.

[...] elaborar uma Agenda com propostas, contemplando soluções e estratégias para potencializar o uso das informações pelos gestores e pela população, nos diversos níveis do SUS, na perspectiva da construção de uma Política Nacional de Informações em Saúde para o país (ABRASCO; GTISP, 2000 p. 1). 
As discussões foram norteadas por eixos temáticos: uso das informações em saúde; tecnologia da Informação; qualidade da Informação e a questão ética relacionada à informação em saúde.

Em relação ao uso das informações em saúde foi destacada a necessidade de inserir o cidadão nos processos de disponibilização de dados, visando o seu empoderamento por meio do acesso às informações de saúde. A democratização do acesso à informação em saúde constituiu a tônica deste debate. Outro aspecto enfatizado foi a baixa utilização das informações no processo de gestão da saúde. Em sua maioria, foi identificado que as decisões tomadas no nível gerencial eram pouco embasadas em informações provenientes dos sistemas de informação de abrangência nacional. Também se verificaram resistências no desenvolvimento de novas formas de gestão, menos verticalizadas e centralizadoras. Neste primeiro eixo (uso das informações em saúde) foi proposto que a informação e tecnologias associadas sejam inseridas na Agenda política de saúde e não restrita aos especialistas.

Para fomentar um amplo processo político em torno da produção, disseminação e uso das informações, torna-se estratégico que a Informação e as tecnologias a ela associadas passem a fazer parte da Agenda Política da Saúde, sob pena desta perder o "bonde da história". Reduzir os desafios que as Tecnologias de Informação vêm representando para as sociedades contemporâneas como questão restrita ao debate dos técnicos e especialistas, em nada contribui para o salto de qualidade que, tanto a gestão da informação quanto a gestão da saúde, estão necessitando no Brasil. Uma questão política é tratada politicamente como sendo "técnica", alijando atores sociais do processo de formulação da Política Nacional de Informação em Saúde (GTISP; ABRASCO, 2000, p. 6).

Reconheceu-se a necessidade da construção de uma política nacional de informações em saúde com participação de diversos atores sociais capaz de superar as resistências e dificuldades já reconhecidas no campo.

Um processo democrático de eleição de prioridades, promovendo uma real mudança de cultura no trato das informações, superando as resistências à inclusão de novos indicadores, novas abordagens, novas tecnologias e novos atores sociais discutindo a Política de Informações em Saúde que se quer para o país (GTISP; ABRASCO, 2000, p. 6).

No que tange à tecnologia da informação (segundo eixo) reconheceu-se que novos modelos de gestão deveriam ser acompanhados de inovações tecnológicas. Entretanto, foi proposto o estabelecimento de padrões devido à fragmentação histórica dos sistemas de informação de abrangência nacional. Este processo de padronização deveria se desenvolver de forma democrática, em três níveis: a padronização do 
vocabulário, a padronização do conteúdo e a padronização dos procedimentos de transmissão da informação. Outros pontos importantes foram propostos: o entendimento de que a padronização não é algo específico de uma instância definidora; a constituição de um prontuário eletrônico do paciente em todos os serviços de saúde compatível com os sistemas de abrangência nacional; implantação do Cartão Nacional de Saúde; o desenvolvimento de critérios de acreditação tanto da informação quanto dos sistemas de informação em saúde. Por fim, destacou-se o ensejo por desenvolver um processo de capacitação amplo envolvendo os profissionais e gestores presentes nas Secretarias Estadual e Municipal de saúde.

Em relação à qualidade da informação em saúde (terceiro eixo) desenvolveu-se o entendimento de que o fluxo informacional, desde a coleta, passando pelo armazenamento e análise, até a disseminação devem ser susceptíveis a procedimentos sistematizados e qualificadores, tais como a validação de dados.

A questão ética relacionada à informação em saúde foi outro eixo norteador dos debates. Os riscos à privacidade dos cidadãos que disponibilizam suas informações nas bases de dados e sistemas de informação em saúde precisam ser considerados e analisados. Assim os padrões de segurança deveriam garantir: a integridade da informação; a privacidade do cidadão e a confidencialidade da informação seguindo o princípio do consentimento livre esclarecido. Propô-se a criação de um Comitê de Ética e Segurança das informações em Saúde com representantes de várias instâncias e da sociedade civil.

O GTISP ainda reafirmou que todos os desafios e proposições destacados nos quatro eixos norteadores da oficina "requerem uma política clara de valorização do processo de produção e disseminação das informações" e também afirmam a necessidade de:

ampliar os recursos financeiros para esta área, tanto para as instituições de serviços de saúde (como o DATASUS, do Ministério da Saúde), em todas as esferas de governo (como os Centros de Informações em Saúde das SES e SMS), quanto para pesquisas e estudos sobre o tema. É importante uma política que fomente a produção de conhecimento nacional nessa área, diminuindo a dependência tecnológica às inovações estrangeiras (ABRASCO; GTISP, 2000, p. 7).

\subsection{As contribuições do GTISP para as versões da PNIIS nos anos de 2003 e 2004}

No VII Congresso Brasileiro de Saúde Coletiva, em Brasília, em 2003, o GTISP se reuniu novamente por meio da oficina de trabalho "Informação em saúde: acertos, erros e perspectivas" (ABRASCO; GTISP, 2003 , s.p.). Foi destacada a necessidade de aprofundar o debate acerca de alguns temas discutidos em anos anteriores, tais como abrangência, cobertura, compatibilização e os sistemas de informação em saúde. A 
política de informação emerge no documento desta oficina como o seu objetivo principal:

Nosso objetivo foi então, reconstruir uma agenda que consubstancie uma política nacional de informações em saúde, enquanto requisito estratégico para avanço do SUS, partindo da análise dos acertos e erros ocorridos na área, na última década (ABRASCO, GTISP, 2003, s.p.).

Nessa oficina os eixos norteadores foram: os sistemas e as metodologias de produção e disseminação de informações em saúde; as questões éticas relacionadas aos sistemas de informação em saúde; a padronização, interoperabilidade e produção de softwares livres; o desenvolvimento de capacitação visando o uso das informações com o enfoque da gestão e planejamento em saúde; o financiamento da incorporação de tecnologias a partir de uma política clara e transparente de investimentos; a estruturação e organização da gestão da informação em saúde. Em relação ao processo de capacitação, o GTISP assumiu o papel de articulador entre as instituições de ensino e pesquisa visando a capacitação por meio de modalidades como a Educação à distância, especialização, mestrados e doutorados.

Também foi proposta pelo GTISP a conformação de uma política de informação integradora, que considere a informação e a tecnologia da informação como indissociáveis, evitando o exclusivismo.

Pretendeu-se na Oficina enfatizar o aspecto da integração. Ou seja, discutir formas de articular todas as iniciativas na área de informações e TIC em Saúde, mais especificamente visando a construção de uma Política Nacional de Informações em Saúde, que enfatize a integração. Consideramos que uma das atitudes mais prejudiciais ao movimento sanitário é o exclusivismo, que não permite uma ampliação do "público", condizente com o seu caráter (ABRASCO; GTISP, 2003, s.p.).

Dando prosseguimento ao percurso histórico do GTISP e suas contribuições para a construção da PNIIS, em 2004, no VI Congresso Brasileiro de Epidemiologia, no Recife, foi realizada a Oficina de Trabalho "A integração necessária - a perspectiva da Epidemiologia na definição de padrões para as Informações em Saúde".

Ao observar os objetivos das duas oficinas realizadas em 2003 e 2004 pelo GTISP percebem-se pequenas diferenças. Na primeira oficina buscava-se "reconstruir uma agenda que consubstancie uma política nacional de informações em saúde, enquanto requisito estratégico para avanço do SUS, partindo da análise dos acertos e erros ocorridos na área, na última década" (ABRASCO; GTISP, 2003, s.p., grifos nossos). Já na segunda oficina empreendia-se em "debater o processo de construção de uma agenda que consubstancie uma política nacional de informações em saúde, enquanto requisito estratégico para 0 
aperfeiçoamento do SUS, a partir da análise dos avanços e recuos ocorridos na área, na última década" (ABRASCO; GTISP, 2004, p. 1, grifos nossos). A quase igualdade dos dois objetivos revela que nestes dois anos o GTISP esforçou-se em (re)construir uma agenda de debates sobre a PNIIS. E, principalmente, entendendo que esta política seria de suma importância para o desenvolvimento do SUS. Exatamente nestes dois anos, de fato, houve um esforço para construção das versões do texto da PNIIS. E o GTISP esteve presente nestes momentos contribuindo com análises, proposições e também debatendo as versões da PNIIS apresentadas.

No ano de 2003 a versão preliminar dos fundamentos da PNIIS foi elaborada pela equipe da área de Informação e Informática do SUS e apresentada aos dirigentes do Ministério da Saúde (MS) (BRASIL, 2003a). Em julho do mesmo ano os dirigentes do MS apresentaram os fundamentos da PNIIS no VII Congresso da ABRASCO.

A partir dos debates e esclarecimentos originou-se a versão 1.1 da PNIIS que foi apresentada à Câmara Técnica da Comissão Intergestores Tripartite, onde contou com as contribuições e o envolvimento do Conselho Nacional de Secretários de Estado de Saúde (CONASS) e o Conselho Nacional de Secretários Municipais de Saúde (CONASEMS) (BRASIL, 2003b). A partir das discussões da versão 1.1 da PNIIS nos municípios e estados, juntamente com outras instâncias, produziu-se a versão 1.2 que foi o centro dos debates do II Seminário Nacional sobre Informação em Saúde da FIOCRUZ, realizado em outubro de 2003, bem como de uma consulta pública ao documento realizada no período de outubro a dezembro de 2003 (BRASIL, 2003c). Após a consulta pública e os reordenamentos do documento, produziu-se a versão 1.3 (BRASIL, 2003d). Essa versão foi então apresentada e discutida na 12a. Conferência Nacional de Saúde. Por fim originou-se a versão 2.0 da PNIIS, em 29 de março de 2004, que incorporou as deliberações definidas anteriormente (BRASIL, 2004).

Além da participação do GTISP nos debates das versões da PNIIS é importante destacar que o grupo contou com participação da pesquisadora Ilara Hammerli Sozzi de Moraes como membro da comissão de sistematização da PNIIS nas versões 1.3 e 2.0. Ressalta-se que a pesquisadora é integrante do GTISP desde sua fundação e se mantém até os dias atuais como coordenadora do grupo.

Apesar dos debates e proposições acerca da PNIIS culminarem na versão 2.0 de março de 2004, o GTISP em junho do mesmo ano, em seu relatório final entende que na "agenda da política nacional de informação em saúde" ainda há algumas questões que precisam ser esclarecidas (ABRASCO; GTISP, 2004 p.2):

Quais opções metodológicas são capazes de efetivar a integração das informações em saúde através da interoperabilidade dos sistemas e da padronização dos dados para propiciar informações em tempo, forma e detalhamento úteis para a gestão do SUS e para o controle social? 
Qual o modelo de gestão da informação em saúde mais adequado para implementar as opções metodológicas a serem definidas?

Quais os conteúdos e modalidades pedagógicas a serem adotados para o processo de educação permanente dos profissionais que atuam no campo da informação e informática em saúde?

Quais as estratégias de fomento de desenvolvimento científico e tecnológico para a tecnologia de informação em saúde?

Quais são os atores e como construirmos a arena para dar continuidade a este debate?

As questões anteriores nortearam os debates da oficina e, sobretudo elaboraram-se recomendações para a agenda da política em construção. Ressalta-se que tanto as questões levantadas como as recomendações configuram-se como contribuições no texto da PNIIS, versão 2.0 (BRASIL, 2004), bem como na versão atual colocada em consulta pública (BRASIL, 2012).

\subsection{0 reconhecimento pelo GTISP das razões (dificuldades) para não implementação da PNIIS}

Em 2006, no âmbito do $11^{\circ}$ Congresso Mundial de Saúde Pública e $8^{\circ}$ Congresso Brasileiro de Saúde Coletiva, desenvolveu-se a oficina de trabalho do GTISP "Pacto pela democratização e qualidade da informação e informática em saúde" (ABRASCO; GTISP, 2006, s.p.). Neste espaço o objetivo foi "analisar a situação atual da Informação e Informática em Saúde e de discutir a construção de um pacto pela democratização e qualidade da Informação e Informática em Saúde". O eixo norteador do debate foi a Política Nacional de Informação e Informática em Saúde (PNIIS) e as dificuldades relacionadas à sua implementação. Segundo o relatório final da oficina a PNIIS "não foi implementada até hoje". Apontaram que é necessário "repor a proposta da PNIIS na mesa de discussão. A Informação em Saúde precisa de uma PNIIS e esta precisa de uma estratégia para ser de fato implementada, não podendo ficar limitada a uma carta de intenções"(ABRASCO; GTISP, 2006, s.p.).

O relatório do GTISP também identificou algumas "razões" para a não implementação da PNIIS (ABRASCO, GTISP, 2006, s.p.):

a)falta de mecanismos do SUS e outros fóruns de discussão para implementar a PNIIS;

Deveriam ter sido mais utilizados mecanismos do SUS (pactuação etc.) para implementar a PNIIS, bem como outros fóruns como, por exemplo, a RIPSA. 
b)falta de investimentos no desenvolvimento de uma inteligência informacional pública, deficiência de diretrizes para aquisição de tecnologias e ampliação do mercado privado no espaço público;

[...] não são feitos investimentos no desenvolvimento de uma inteligência informacional pública, havendo um forte aumento das terceirizações, seja no desenvolvimento de software, seja na manutenção do "setor de informática".

Na ausência de diretrizes para a busca de soluções para seus problemas de Informação e Informática, os gestores implementam de forma isolada as soluções que thes são oferecidas pelo mercado.

c)desintegração dos sistemas de informação nacionais;

[...] ainda se está longe da desejada integração dos Sistemas de Informações Nacionais, que também deveriam abranger os serviços de saúde, públicos e privados, de todo o país. Além disso, seria desejável que houvesse a articulação dos inquéritos de base nacional com os SIS (Sistemas de Informação em Saúde), validando ou não os sistemas de informação nacionais. No entanto, aparentemente, neste momento, nem o suplemento de saúde da PNAD tem sua periodicidade garantida, havendo a necessidade de maior transparência e mais debate sobre a otimização de amostras e sobre os inquéritos nacionais.

d)desenvolvimento de sistemas ainda sob a forma tradicional do controle e centralização;

[...] no Ministério da Saúde, como se verifica há anos, os sistemas continuam sendo desenvolvidos de forma centralizada, verticalizada, fragmentada e proprietária, em vez de se buscar a padronização e interoperabilidade ("conversa entre sistemas"). Isso ocorre mesmo nos sistemas mais novos, como por exemplo, o SISMAMA.

Os sistemas focalizam o controle, em vez de serem construídos a partir do processo de trabalho, redundando em aumento da carga de trabalho por meio da criação de mais processos de controle.

e)iniciativas insuficientes de interoperabilidade e padronização;

É desejável, portanto, que as informações sobre a saúde suplementar tenham interoperabilidade com o setor público e que sejam estabelecidos limites para o uso dos dados, respeitando preceitos éticos, que garantam a privacidade do cidadão brasileiro. 
$\mathrm{Na}$ oficina foram discutidas três temáticas: a necessidade de investimentos em tecnologia da informação, bem como a sua regulação; as questões éticas relacionadas à informação em saúde e o Pacto pela Saúde 2006 (ABRASCO; GTISP, 2006). Estas temáticas foram elencadas e debatidas por se acreditar na sua potencialidade em suplantar as razões (dificuldades) para não implementação da PNIIS.

$\mathrm{Na}$ primeira temática a discussão central foi pautada no entendimento de que os setores de informação e informática em saúde dos Estados e União precisam ser empoderados no sentido de serem os responsáveis pelo desenvolvimento da informação e informática em saúde. Neste sentido, o setor privado seria apenas complementar ao setor público. As terceirizações foram alvo de debates e consideradas como prejudiciais ao setor público. Considerou-se a necessidade de ampliar os investimentos nos estados e municípios visando à formação e capacitação de recursos humanos em informação e informática em saúde.

Em relação à questão ética "O Brasil pouco avançou na criação de salvaguardas éticas e jurídicas que garantam a segurança da inviolabilidade da identificação do cidadão em qualquer base de dados existente" (ABRASCO; GTISP, 2006, s.p.). Três aspectos éticos devem ser garantidos: a integridade da informação; a privacidade do cidadão e a confidencialidade da informação. Ainda considerou-se que a troca de informações entre a esfera pública e privada não garantem a privacidade das informações dos cidadãos. Na tentativa de solucionar esta questão ética, foi sugerido que a ABRASCO e a RIPSA participem deste processo de diálogo e que seja criado um comitê responsável por desenvolver princípios e estratégias no que diz respeito à privacidade das informações dos cidadãos.

Outra questão debatida foi o Pacto pela Saúde 2006. Os participantes entenderam que a informação e tecnologias correlatas são fundamentais para o cumprimento do pacto. Entretanto, identificou-se que o pacto estava sendo construído a partir de uma ótica onde a informação serviria apenas para o controle, e não para os processos de gestão e planejamento. Em síntese, o pacto limitava-se em manter a operacionalização dos sistemas de informação de abrangência nacional. Como resultado, persistiria o mesmo modelo de gestão da informação, focado na centralização, verticalização e o controle. Para solucionar as questões do pacto, considerou-se a necessidade de que fosse estabelecido em relação à informação: o que deveria ser pactuado; as competências e atribuições relacionadas aos sistemas de informação; a determinação das informações que devem fazer parte desses sistemas.

\subsection{O GTISP na elaboração de planos diretores visando suplantar os desafios relacionados à definição e implementação da PNIIS}

Em julho de 2007 o GTISP completou 15 anos de existência. Neste período os vários problemas relacionados ao campo da informação e 
informática em saúde, bem como dificuldades na implementação da PNIIS persistiam, o que levou o GTISP a delinear planos diretores com vistas a definir metas e traçar estratégias numa perspectiva analítica e de intervenção.

Assim, o GTISP promoveu a oficina de trabalho "Construindo o Plano Diretor para o Desenvolvimento da Área de Informação e Informática em Saúde" no âmbito do IV Congresso Brasileiro de Ciências Sociais e Humanas em Saúde, em Salvador. Os debates propiciaram a elaboração do I Plano Diretor para o Desenvolvimento da Informação e Tecnologia da Informação em Saúde (2008-2012) (I PlaDITIS) (ABRASCO; GTISP, 2008). No que diz respeito à PNIIS o GTISP reafirmou o seu propósito:

[...] o GTISP vem procurando subsidiar a formulação e implementação de uma Política Nacional de Informação e Informática em Saúde voltada para a defesa do direito universal à saúde, do dever do Estado em garantir esse direito e da democratização e qualidade das informações em saúde, inserida em um amplo processo de inclusão digital dos diferentes segmentos da sociedade (ABRASCO; GTISP, 2008, p. 1).

A elaboração do I PlaDITIS buscou contemplar três dimensões consideradas estratégicas: o ensino, a pesquisa e a informação juntamente com suas tecnologias na gestão, regulação, programas e serviços de saúde. Ainda possui três marcos referenciais que norteiam suas ações. No primeiro, a informação em saúde é um dever do Estado e um direito de todos, devendo estar a serviço da sociedade, da justiça social e da democracia. O segundo marco é definido pelo entendimento de que a informação em saúde é um bem público e passível de ser gerido por mecanismos colegiados, democráticos e expostos ao exercício do controle social do SUS. Por fim, o terceiro marco defende o posicionamento de que as Tecnologias da Informação devem ser tratadas como um componente da PNIIS, não devendo ser restrita aos especialistas e muito menos ser tratada como porte de um processo de incorporação tecnológico.

$\mathrm{Na}$ oficina os participantes reconheceram que ainda permaneciam grandes desafios relacionados ao campo da informação e informática em saúde. Especificamente, em relação à PNIIS os desafios estavam relacionados a:

a) conflitos pela direcionalidade da PNIIS atrelada ao mercado e a terceirização, bem como sucateamento das instâncias públicas.

Forte pressão das empresas privadas de informática em disputa pelo setor público da saúde, enquanto fatia substantiva de mercado, onde há uma luta pela direcionalidade da Política de Informação e Informática em Saúde. Em paralelo, observa-se um processo de sucateamento das instâncias públicas de gestão da informação e informática em saúde, fortalecendo a tendência pela opção à terceirização 
sem critérios técnicos transparentes e com controle escasso dos serviços terceirizados (ABRASCO; GTISP, 2008, p. 4).

b)ausência de coordenação da política institucional da informação.

De fato, observa-se a ausência de uma coordenação da política institucional da informação e suas tecnologias, o que favorece a manutenção de feudos técnicos estanques, sistemas fragmentados e verticalizados, interesses privados face à possibilidade de vendas de multiplicidade de "soluções de informática" (ABRASCO; GTISP, 2008 p.11-12).

c)falta de apoio político para aprovação de uma política de informação em saúde

Como mudar tal quadro? Uma abordagem mais direta recomenda a mobilização de esforços para promover apoio político ao fortalecimento da gestão da informação. Esse apoio se consubstancia na aprovação de uma política nacional construída coletivamente e pactuada pelos envolvidos, que pressuponha o uso racional de tecnologias de informação, simplificando processos de coleta e possibilitando a interoperabilidade dos sistemas atuais, com acesso aos vários segmentos interessados (ABRASCO; GTISP, 2008 p.20).

Definiu-se nove eixos temáticos em debate no I PlaDITIS. Em cada eixo elencou-se algumas metas e propostas que deveriam ser operacionalizadas e avaliadas no quinquênio (2008 e 2012). Em relação à PNIIS definiu-se o eixo temático:

9)Avaliação da Política Nacional de Informação e Tecnologia de Informação em Saúde

Parece-nos que esta é uma proposta muito próxima da missão do GTISP por constituir-se um Grupo da ABRASCO plural e autônomo. O processo avaliativo de políticas públicas é complexo, em especial da Política de Informação e Informática em Saúde, do Plano Nacional de Saúde e do Pacto pela Saúde, necessitando de diversidade metodológica e amplitude de atuação tendo como objeto tanto as três esferas de governo, quanto as Agências reguladoras (ABRASCO; GTISP, 2008, p. 23).

A partir deste eixo temático foram propostas algumas metas para o período compreendido entre os anos de 2008 e 2012:

Constituição de mecanismos autônomos de avaliação da Política Nacional de Informação e Informática em Saúde implementados por instituições de pesquisa em saúde 
vocacionadas para a área de informação e tecnologias de informação. Até 2010.

Prioridade na convocação da I Conferência Nacional de Saúde de Informação, Informática e Comunicação em Saúde. 2008 / 2009. (ABRASCO; GTISP, 2008, p. 23).

Em novembro de 2012, no X Congresso Brasileiro de Saúde Coletiva, em Porto Alegre, o GTISP se reuniu novamente durante a oficina de trabalho: "Plano Diretor para o desenvolvimento da Informação e Tecnologia de Informação em Saúde - PlaDITIS 2013-2017 - no contexto da atual governança de informação e de implantação do Registro Eletrônico em Saúde/RES" (ABRASCO; GTISP, 2013). Dentre os marcos referenciais a Política de informação é citada, mas como uma questão ainda não resolvida apesar dos esforços empreendidos.

A história brasileira da informação e da tecnologia de informação em saúde (ITIS), os estudos sobre as experiências de implantação dos sistemas de informações de saúde de base nacional, as diversas iniciativas do Cartão Nacional de Saúde desde 1999, os diferentes esforços de pactuação de uma Política Nacional de Informação e Informática em Saúde e a constituição do Comitê de Informação e Informática em Saúde (CIINFO) são exemplos de algumas referências a serem levadas em conta por suscitarem velhas questões ainda não resolvidas e questões novas que requerem respostas novas para a melhoria das ITIS no Brasil. (ABRASCO; GTISP, 2013, p. 9).

O norteamento das discussões deu-se a partir de cinco dimensões estratégicas para o desenvolvimento da informação e tecnologias de informação em saúde no Brasil.

Em relação à primeira dimensão estratégica foram debatidas as seguintes questões: o papel do Estado e a relação público-privado; governança e modelo de gestão da ITIS; responsabilidades da gestão da ITIS; definição de padrões e certificação de sistemas de informações/ehealth. Neste contexto enfatizou-se que o setor da saúde tem se tornado um mercado promissor para as soluções tecnológicas. Empresas privadas aliadas aos interesses políticos, de consultores e de outras esferas utilizam-se do espaço público, considerado ineficiente, para implementar os seus projetos hegemônicos visando predominantemente o lucro. Subordinam as políticas públicas aos seus interesses relacionados ao capital.

A depender do espaço e tempo, observa-se um 'aprisionamento' de algumas estruturas de ITIS no interior de um triângulo de ferro dos interesses de parlamentares; 
burocratas, consultores e cargos de confiança que circulam suas carreiras entre o governo e o mercado, e membros gestores do executivo cujas 'campanhas' foram apoiadas por estas empresas. Em outras palavras, manifesta-se a subordinação da política pública aos interesses da fração hegemônica do capital em sua reprodução no setor saúde (ABRASCO; GTISP, 2013, p. 12).

Diante dos conglomerados empresariais que se formam em torno da TI em Saúde, é preciso atenção nas articulações que se formam entre o público e o privado para que não representem uma canalização dos interesses privados para o interior da res pública, reduzindo a função do SUS a um 'rico mercado de contratos'. (ABRASCO; GTISP, 2013, p. 13).

A política centrada na terceirização da ITIS ancora-se no pensamento que considera o Estado brasileiro, mais especificamente o Ministério da Saúde através do DATASUS, como portador da ineficiência e da ineficácia e que a excelência e inovação são encontradas somente no mercado das empresas de tecnologia da informação (TI) (ABRASCO; GTISP, 2013, p. 16).

Algumas propostas emergiram a partir do debate sobre a primeira dimensão estratégica "governança e gestão da informação e tecnologia de informação em saúde". As propostas, de forma geral, enfatizam a necessidade de empoderar o setor público para desenvolver tecnologias, capacitar recursos humanos e renovar o quadro de especialistas. Também foi proposta a necessidade de definição dos limites de atuação do setor privado no espaço público. Para esta definição é necessário a participação coletiva envolvendo gestores, profissionais de saúde, conselhos de saúde e instituições de pesquisa ciência e tecnologia.

A segunda dimensão estratégica contemplada no II PlaDITIS foi "Pesquisa, Desenvolvimento e Inovação em Informação e Tecnologia de Informação em Saúde". Neste momento identificou-se que a área de Informação e Tecnologia de Informação em Saúde (ITIS) não está recebendo a atenção da comunidade científica da saúde coletiva. Outro aspecto abordado é que a justificativa da 'transferência tecnológica' tem proporcionado a alocação de recursos nas terceirizações de ITIS. As propostas em destaque estão centradas na necessidade de uma "Política de Estado voltada para a constituição de equipes de excelência em trabalho colaborativo de forma gradual, nos moldes de desenvolvimentos translacionais e que leve em conta a interdisciplinaridade" (ABRASCO; GTISP, 2013, p. 22-23). 
O "Ensino e formação permanente de equipes de informação e tecnologia de informação em saúde" foi definido como a terceira dimensão estratégica do II PlaDITIS. O debate pautou-se pelo entendimento de que educação e formação profissional são investimentos sociais e político. Neste sentido três elementos foram apontados como estruturantes de um processo consistente de formação permanente de equipes de ITIS, são eles: "vontade política, liderança institucional e redes de cooperação entre centros formadores" (ABRASCO; GTISP, 2013, p. 24).

A quarta dimensão estratégica foi "Ética, privacidade e confidencialidade". Neste momento buscou-se discutir o possível monitoramento da vida do cidadão e a falta de um controle social e ético. O "limite político entre a esfera pública e a esfera privada da vida" foi resgatado como uma questão dilemática.

As novas tecnologias de informação tornam possíveis, cada vez mais, o controle da vida do cidadão e permitem seu rastreamento em diferentes bases de dados. Impõe-se, portanto, discutir o limite político entre a esfera pública e a esfera privada da vida e as estratégias de uso das tecnologias de informação e comunicação de massa nas sociedades globalizadas (ABRASCO; GTISP, 2013, p. 26).

Também se questionou sobre o fato do desconhecimento dos interesses das políticas de e-gov em desenvolvimento no país. Assim foi questionado: "as políticas de governo eletrônico (e-gov) estão subordinadas a que interesses? Será necessário cotejar a privacidade/confidencialidade com as políticas de governo eletrônico" (ABRASCO; GTISP, 2013, p. 26). Foi proposta a necessidade de se instituir um arcabouço jurídico-ético em defesa da privacidade e confidencialidade dos dados do cidadão. Entretanto este debate deve ser coletivo envolvendo várias instâncias e os mais interessados, a população. Também foi proposta a necessidade de desenvolver estudos sobre a viabilidade de aplicação do Termo de Consentimento Livre e Esclarecido aos usuários quando das coletas de suas informações. Por fim, apontou-se a necessidade de desenvolver a capacitação dos profissionais do SUS sobre o respeito, confidencialidade, ética e privacidade dos dados coletados e ainda a definição de penalidades para empresas que violarem o princípio da privacidade e confidencialidade.

A quinta, e última dimensão estratégica estabelecida no II PlaDITIS, foi "Informação e tecnologia da informação em saúde: Democracia, controle social e justiça cognitiva". Debateu-se a necessidade da participação da população no controle social sobre as ações do Estado. Para isso a necessidade de Democratização do acesso às informações e TI em saúde. Trabalhou-se com o entendimento de que a democratização do acesso à informação e TI em saúde ocorre quando: 
1) Os cidadãos têm a garantia de acesso às informações coletadas pelo Estado sobre a sociedade; 2) O Estado torna-se transparente para a sociedade, em seus diferentes dispositivos; e 3) São estabelecidos mecanismos de participação direta de representantes da sociedade no próprio processo de definição da política pública relacionada à informação e TI em saúde. (ABRASCO; GTISP, 2013, p. 27).

O debate ainda apontou que as ITIS não são utilizadas pra o benefício da ampliação da potência de controle social sobre as ações do Estado. Também se destacou que existe, no país, uma cultura política que:

[...] alija representantes da sociedade desse debate sob a alegação de tratar-se de uma questão "técnica", afeta aos especialistas. Constitui-se uma racionalidade tecnocrática para, politicamente, afastar o cidadão do debate de uma política pública que incide sobre o projeto de democracia e saúde que a sociedade pretende construir no país (ABRASCO; GTISP, 2013, p. 27).

As propostas que emanaram das discussões estiveram, de forma geral, atreladas à necessidade de disseminar a informação em saúde em linguagem inteligível para representantes dos usuários e conselheiros, promovendo a consciência sanitária.

\section{Discussões dos resultados}

Os resultados anteriores apresentam o panorama dos discursos dos componentes do GTISP, e de forma geral concentram o debate sobre: a valorização do entono sócio-cultural que envolve o campo da informação e informática em saúde, bem como a política de informação; a necessidade de construir uma política de informação para a área de forma coletiva, plural, pactuada e gerida de forma colegiada; o resgate da informação como um direito de todos e a necessidade de romper com o modelo de gestão da informação hegemônico no Brasil; a definição de planos diretores com vistas à suplantar as dificuldades reconhecidas no campo da informação e informática em saúde.

Primeiramente, o GTISP apesar de debater e propor estratégias sobre os aspectos tecnológicos que envolvem uma política de informação vai além ao reconhecer que aspectos como a cultura, os recursos humanos, a qualidade da informação, a ética, o financiamento, a estrutura pública e suas relações com o privado, também são aspectos a serem considerados. Para Aun (1999) há um entorno sócio cultural envolvendo uma política de informação nacional e isto se configura como um desafio a ser considerado, principalmente no contexto atual de uma sociedade global da informação. Neste contexto, destaca que a construção de uma 
política de informação não pode ser empobrecida ao focar apenas nos aspectos tecnológicos e legislações. Mas o ambiente social e humano também devem serem considerados na política em construção. Marques e Kerr Pinheiro (2011) declaram que os estudos das políticas de informação podem auxiliar na apreensão de um contexto marcado pela confluência de fatores sociais, econômicos e tecnológicos. Assim, uma política de informação, seja qual for o seu subdomínio, como o da saúde, deve ser norteada para além do imperativo tecnológico.

Um segundo aspecto que emerge dos discursos do GTISP é o entendimento de que é preciso construir uma política de informação de forma coletiva, valorizando a pluralidade dos sujeitos envolvidos, a representatividade de suas instâncias, bem como a sua gestão colegiada. Para Higino et al (2008) o processo de construção de políticas de informação envolve a complexidade de considerar a participação de múltiplos atores. Esta é uma condição sine qua non que envolve certa heterogeneidade associada ao debate sobre tecnologia, informação, as pessoas e suas interações sociais. Moraes e Vasconcellos (2005) ao considerar a necessidade de múltiplos atores na construção de uma política de informação, apontam a necessidade de envolver representantes da população neste processo. Ainda afirmam que é preciso exercitar a democracia participativa, onde os debates e decisões coletivas devem ser incentivados e respeitados. Entretanto, o discurso da coletividade, pluralidade e gestão colegiada envolve o reconhecimento de conflitos que estão no bojo das relações de poder. Para Marques e Kerr Pinheiro (2011) a relação entre informação e poder é um desafio que emerge mediante a construção de uma política de informação. Há várias possibilidades de manifestação dos efeitos de poder e em vários espaços sociais. A discussão da abertura do espaço público ao mercado privado é um marco conflitante na construção de políticas de informação, que elucida a presença de relações de poder. Para Bobbio (1995) a própria discussão conceitual de política remete ao poder que se manifesta nos campos econômico, ideológico e político. Neste sentido Braman (2004) afirma que há relações de poder que se estabelecem no campo da política de informação, e atrelado ao regime político vigente constituído por regras, leis e normas advindas das relações complexas entre atores formais e informais que constituem o Estado. É preciso reconhecer as relações de poder neste espaço da política e de seu regime. No contexto da área da saúde, mais especificamente no campo da informação e informática em saúde, as relações de poder emergem, também, a partir da relação entre o público e o privado. (MORAES, 2002; CAVALCANTE; KERR PINHEIRO, 2011). Neste sentido, o GTISP avança ao reconhecer a atuação do privado no meio público e reforça a necessidade de que o espaço público seja estruturado para exercer as ações relacionadas ao campo da informação e informática em saúde, tendo a PNIIS que normatizar este processo. 0 GTISP não nega a impossibilidade da atuação do mercado privado no espaço público, mas busca que a regulação desta atuação aconteça. E ainda, entende que o espaço público representado pelas instâncias nas 
esferas federal, estadual e municipal sejam equipados, estruturados e organizados para atuarem na área da informação e informática em saúde. O desafio está em equilibrar a oferta de serviços de informação e informática em saúde pelo espaço público e a contenção da terceirização excessiva empreendida pela indústria de tecnologias da informação. Neste embate, a definição de uma política de informação em saúde ganha relevância visto a sua potencialidade em legislar sobre a área. Entretanto, é preciso garantir a sua efetivação em todas as esferas do SUS. Ressaltase que esta política antes de ser aplicável precisa ser conhecida por todos e pactuada entre os gestores. Neste sentido, a sua construção não deve ocorrer a partir de uma pluralidade apenas em uma esfera de gestão, mas envolver os profissionais e a população que estão no locus assistencial. Assim, é possível a emissão de efeitos de poder em várias esferas e contribuir para avanços na regulação e fiscalização do campo da informação e informática em saúde.

Reconhece-se o esforço do GTISP em propor os elementos componentes da pactuação. Entretanto, a construção de uma política de informação reguladora deste campo é uma decisão política e necessita ser reconhecida, legitimada e praticada, no caso do SUS, pelas três esferas de gestão (federal, estadual e municipal). A PNIIS a ser pactuada não deve ser uma decisão isolada de apenas uma ou outra instância, ou um esforço de um grupo isolado. É certo também que a pactuação da PNIIS, para ser realizada e cumprida, precisa ser objeto de conhecimento e debate das instâncias e pessoas que compõem as esferas de gestão, pois do contrário a PNIIS será apenas mais uma norma imposta e sem sentido em seu cumprimento, principalmente para gestores e profissionais que estão nos níveis mais locais da assistência a saúde. Ainda, é importante ressaltar que a pactuação da PNIIS também deve levar em conta as possibilidades de seu cumprimento nas esferas de gestão. Pactuar o que não pode ser cumprido pela falta de infraestrutura, falta de recursos humanos, financiamento insuficiente, dentre outros, é expor uma política ao seu insucesso. E sabe-se que no Brasil as instâncias públicas relacionadas ao campo da informação e informática em saúde, em sua maioria, ainda carecem de estruturação (MORAES, 2002; ABRASCO; GTISP, 2008).

Outro aspecto que emerge dos debates do GTISP foi 0 reconhecimento de um direito conquistado ainda na Constituição brasileira de 1988: o acesso à informação é um direito de todos e um dever do Estado. Isto impulsionou a necessidade de uma nova conformação para a gestão da informação em saúde no Brasil e, por conseguinte, direcionou os marcos referencias da política de informação em construção. No Brasil, Branco (2006) demonstrou que o modelo de gerenciar a informação foi, durante muitos anos, centrado no autoritarismo do Estado e seu ensejo pelo controle. Gruman (2012) endossa essa afirmação quando declara que as questões envolvendo a necessidade do sigilo sempre foram utilizadas no Brasil como justificativa para tratar a informação como posse irrestrita de governo. Assim, o acesso à informação para todos e a transparência foram alijados dos interesses governamentais. O posicionamento do 
GTISP em resgatar o direito de acesso à informação e a necessidade da democratização da informação rompe com a perspectiva de um modelo centrado no controle, onde "o Estado tem o direito de perguntar aos indivíduos [...] e o indivíduo tem o dever de responder" (MORAES; VASCONCELLOS, 2005, p. 88). Nesta reflexão a busca é inverter o modelo hegemônico, atribuindo ao cidadão a possibilidade de exercer o seu papel ativo dentro da sociedade, de perguntar, questionar e consentir em disseminar as suas próprias informações.

Apesar do GTISP resgatar o debate sobre o direito a informação em saúde e incentivar a democratização do acesso a informação, ainda é preciso avançar. Para Gruman (2012) e Batista (2012) a conquista do direito à informação e o estabelecimento de uma política de informação devem acontecer de forma paralela. Ainda reforçam que tanto o direito à informação, bem como a política devem ser conhecidos por todos. É preciso promover condições para que a população se aproprie da sua existência, reconheça o seu direito, exija e estabeleça o seu papel de cidadania. A lei, a política de informação e as condições necessárias para o controle social são igualmente importantes para que o direito à informação em saúde se estabeleça de fato. Neste sentido está a necessidade de "ampliar e popularizar os meios de distribuição da informação; expandir a rede de disseminação; e desenvolver políticas e ações de disseminação de informações em saúde voltadas para o controle social" (LIMA et al., 2009, p. 124). Aqui, o acesso à informação e a sua democratização ganham sentido, pois a necessidade da informação é com vistas à participação social, ao engajamento, à avaliação dos serviços de saúde e à transformação da realidade. Enfim, o debate empreendido sobre a necessidade do acesso à informação também precisa avançar no sentido de estímulo do controle social para a transformação do contexto social e a redução das iniquidades. Não basta a existência do direito à informação, da disponibilidade do acesso, e de uma política, se as instâncias responsáveis pelo controle social assim como a população não se apropriam dos mesmos e não os utilizam. Estabelece-se o desafio de mobilizar o corpus social para o exercício da cidadania com vistas à justiça social.

A partir dos anos 2000 o GTISP propôs a criação de Planos Diretores para o Desenvolvimento da Informação e Tecnologia de Informação em Saúde (PlaDITIS) com vistas à suplantar as dificuldades reconhecidas no campo da informação e informática em Saúde. Os PlaDITIS proporcionaram a análise do contexto nacional acerca do campo da informação e informática em saúde, principalmente reconhecendo as dificuldades existentes, bem como propondo estratégias de solução. Estes planos têm contribuído no reconhecimento de desafios a serem superados, bem como têm destacado elementos a serem compreendidos, incorporados e normatizados pela política de informação em construção. É importante ressaltar que os PlaDITIS mantêm as reflexões empreendidas pelo GTISP ao longo de quase 20 anos de sua existência, bem como representam o amadurecimento deste grupo neste constructo histórico. 
Considera-se o esforço do GTISP na criação dos PlaDITIS com o objetivo de intervir sobre o campo da informação e informática em saúde, porém alguns autores defendem que os planos são temporais, não são legislados e não implicam na participação dos poderes executivos e legislativo. A política de informação, pelo contrário, exige um posicionamento do governo na definição de normas, leis e reconhecimento em todo o território. Além de possuir uma temporalidade de longo prazo (MOORE, 1997; AUN 2003; MARQUES; KERR PINHEIRO, 2011). Para Kerr Pinheiro (2010) mesmo quando o imperativo tecnológico é suplantado em detrimento da valorização do conteúdo, ainda há um determinismo ancorado no discurso da inovação que consegue submeter a política de informação e sua comunicação por meio de planos ou programas. A autora ainda considera que há um imediatismo nos planos que "dificulta o reconhecimento das políticas como processos de mediação e de tradução" (KERR PINHEIRO, 2010, p. 115).

Entretanto, acreditamos que os PlaDITIS, como uma formulação não governamental, mas coletiva e plural, não substitui a PNIIS em construção, pelo contrário, a reforça e exige a sua legitimação no cenário nacional. Endossando esta afirmação o próprio GTISP aponta o PlaDITIS como o resultado de um esforço que buscou construir uma "Agenda que consubstancie a formulação, implementação e avaliação da Política Nacional de Informação e Informática em Saúde, enquanto requisito estratégico para o aperfeiçoamento do SUS" (ABRASCO; GTISP, 2008, p. 3). Além disso, a sua formulação configurou-se como um espaço de construção coletiva e de adensamento do conteúdo teórico relacionado à construção desta política. Doravante, a busca é pela efetivação de uma política de informação que vem sendo debatida, construída, mas ainda não legislada.

\section{Considerações finais}

A partir da evolução histórica da atuação do GTISP e seu amadurecimento verificou-se que o mesmo tem se conformado como um espaço de contribuições para o campo da informação e informática em saúde, bem como para a política de informação em construção. O grupo no decorrer da história buscou discutir temáticas em uma perspectiva plural, participativa e propôs estratégias inovadoras para os desafios emergentes. Neste sentido também contribuiu com a construção da política de informação em saúde ao propor o rompimento com um modelo político característico de um período marcante da história brasileira. 0 período ditatorial deixou heranças para a atual práxis informacional em saúde: a centralização da informação, a informação para o controle, a assimetria da informação e o sigilo do Estado justificando o modelo de gestão da informação. O GTISP no decorrer da história proporcionou o adensamento de reflexões e proposições compatíveis com a política de informação que se deseja estabelecer e legitimar no espaço social, bem como injetou um debate que vai além do imperativo tecnológico. Remete 
à necessidade de valorizar, nesta política, os aspectos socioculturais que envolvem a informação em saúde no contexto do SUS e suas organizações, bem como o próprio regime informacional que a constitui.

Entretanto, ainda é preciso considerar a existência de um embate e certa morosidade, na formalização, normatização e publicização da PNIIS nas esferas do SUS. Ao longo de quase quatro décadas a construção de uma política de informação em saúde tem se desenvolvido, porém esta construção tem se constituído na elaboração de textos (versões), responsabilização de instâncias e promulgação de portarias e resoluções. É preciso avançar no sentido de legitimar definitivamente a PNIIS e não apenas alguns de seus elementos. Por fim, a PNIIS, como uma elaboração plural e política também precisa se efetivar no lócus assistencial.

\section{Referências}

APPOLINÁRIO, F. Dicionário de metodologia científica: um guia para a produção do conhecimento científico. São Paulo: Atlas, 2009.

ASSOCIAÇÃO BRASILEIRA DE PÓS-GRADUAÇÃO EM SAÚDE COLETIVA (ABRASCO); Grupo Técnico de Informação em Saúde e População (GTISP) (Org.). Informação em saúde a serviço da sociedade. Rio de Janeiro, 1993. $18 \mathrm{p}$.

ASSOCIAÇÃO BRASILEIRA DE PÓS-GRADUAÇÃO EM SAÚDE COLETIVA (ABRASCO); Grupo Técnico de Informação em Saúde e População (GTISP) (Org.). Utilização de grandes bancos de dados nacionais. Belo Horizonte, 1994. 7 p.

ASSOCIAÇÃO BRASILEIRA DE PÓS-GRADUAÇÃO EM SAÚDE COLETIVA (ABRASCO); Grupo Técnico de Informação em Saúde e População (GTISP) (Org.). Compatibilização de bases de dados nacionais. Águas de Lindóia, 1997. 2 p.

ASSOCIAÇÃO BRASILEIRA DE PÓS-GRADUAÇÃO EM SAÚDE COLETIVA (ABRASCO); Grupo Técnico de Informação em Saúde e População (GTISP) (Org.). Informações para a gestão do SUS: necessidades e perspectivas. Salvador, 2000. 10 p.

ASSOCIAÇÃO BRASILEIRA DE PÓS-GRADUAÇÃO EM SAÚDE COLETIVA (ABRASCO); Grupo Técnico de Informação em Saúde e População (GTISP) (Org.). Informação em Saúde: acertos, erros e perspectivas. Brasília, 2003. 4 p.

ASSOCIAÇÃO BRASILEIRA DE PÓS-GRADUAÇÃO EM SAÚDE COLETIVA (ABRASCO); Grupo Técnico de Informação em Saúde e População (GTISP) (Org.). A integração necessária: a perspectiva da epidemiologia na definição de padrões para as informações em saúde. Recife, 2004. 4 p.

ASSOCIAÇÃO BRASILEIRA DE PÓS-GRADUAÇÃO EM SAÚDE COLETIVA (ABRASCO); Grupo Técnico de Informação em Saúde e População (GTISP) (Org.). Pacto pela democratização e qualidade da informação e informática em saúde. Rio de Janeiro, 2006. 7 p. 
ASSOCIAÇÃO BRASILEIRA DE PÓS-GRADUAÇÃO EM SAÚDE COLETIVA (ABRASCO). Grupo Técnico de Informação em Saúde e População (GTISP) (Org.). Plano Diretor de Informação e Tecnologia de Informação em Saúde: 2008-2012. Brasília: ABRASCO, 2008. Disponível em: <http://www.abrasco.org.br/grupos/arquivos/20100210132738.pdf>. Acesso em: 15 mar. 2009.

ASSOCIAÇÃO BRASILEIRA DE PÓS-GRADUAÇÃO EM SAÚDE COLETIVA (ABRASCO). Grupo Técnico de Informação em Saúde e População - GTISP (Org.). Plano Diretor para o desenvolvimento da Informação e Tecnologia de Informação em Saúde - PlaDITIS 2013-2017: no contexto da atual governança de informação e de implantação do Registro Eletrônico em Saúde/RES. Brasília: ABRASCO, 2013.

AUN, M. P. A construção de políticas nacional e supranacional de informação: desafio para os Estados nacionais e blocos regionais. Ciência da Informação, Brasília, v. 28, n. 2, p. 115-123, mai/ago.1999.

AUN, M. P. Políticas públicas de informação e desenvolvimento. In: PAIM, I. (Org.). A gestão da informação e do conhecimento. Belo Horizonte: ECI/UFMG. 2003. v.1. p. 55-90.

BATISTA, C. L. Informação pública: controle, segredo e direito de acesso. Intexto, Porto Alegre, UFRGS, n. 26, p. 204-222, jul. 2012.

BOBBIO, N. Política. In: BOBBIO, N. (Org.). Dicionário de política. Brasília: Universidade de Brasília, 1995. p. 954-963.

BRAMAN, S. The emergente global information policy regime. In: BRAMAN, S. (Ed.). The emergent global information policy regime. Houndsmills, UK: Palgrave Macmillan, 2004. p. 12-38.

BRANCO, M. A. F. Informação em saúde: uma ciência e suas políticas em uma nova era. Rio de Janeiro: Editora Fiocruz, 2006.

BRASIL, Ministério da Saúde. Portaria no 3.947, de 25 de novembro de 1998. Aprova os atributos comuns a serem adotados, obrigatoriamente, por todos os sistemas e bases de dados do Ministério da Saúde, a partir de $1^{0}$ de janeiro de 1999. Diário Oficial da República Federativa do Brasil, Brasília, 14 jan. 1999. Seção 1, p. 8. Disponível em: <http://bvsms.saude.gov.br/bvs/saudelegis/gm/2011/prt2072_31_08_20 11.html>. Acesso em: 25 abril 2012.

BRASIL. Ministério da Saúde. A construção da Política de Informação e Informática em Saúde do SUS. Versão 1.0. Brasília: Ministério da Saúde, 2003a. 20 p.

BRASIL. Ministério da Saúde. A construção da Política de Informação e Informática em Saúde do SUS. Versão 1.1. Brasília: Ministério da Saúde, 2003b. 21 p.

BRASIL. Ministério da Saúde. A construção da Política de Informação e Informática em Saúde do SUS. Versão 1.2. Brasília: Ministério da Saúde, 2003c. 22 p. 
BRASIL. Ministério da Saúde. A construção da Política de Informação e Informática em Saúde do SUS. Versão 1.3. Brasília: Ministério da Saúde, 2003d. 22 p.

BRASIL. Ministério da Saúde. Política Nacional de Informação e Informática em Saúde. Versão 2.0. Brasília: Ministério da Saúde, 2004. 38 p.

BRASIL. Ministério da Saúde. Comitê de Informação e Informática em Saúde (CIINFO). Política Nacional de Informação e Informática em Saúde. Brasília: Ministério da Saúde, 2012. 38 p.

CAVALCANTE, R. B.; KERR PINHEIRO, M. M. Política Nacional de Informação e Informática em Saúde: avanços e limites atuais. Perspectivas em Gestão \& Conhecimento, v. 1, n. 2, p. 106-119, jul./dez. 2011.

CELLARD, A. A análise documental. In: POUPART, J. et al. A pesquisa qualitativa: enfoques epistemológicos e metodológicos. Petrópolis: Vozes, 2008.

GRUMAN M. Lei de acesso à informação: notas e um breve exemplo. Revista Debates, n. 6, p. 97-108, 2012.

HIGINO, A.F.F.; ARAÚJO, R.F.; SCOTT, C.S.P. Construção de políticas de informação: aspectos epistemológicos e metodológicos. Liinc em Revista Online, v. 4, n. 2, p. 286-302, set. 2008.

KERR PINHEIRO, M. M. L'État Informationnel et les changements dans les formes de médiations info-communicationnelles des connaissances sur les politiques de l'information. Recherche post-doctorale en Sciences de I'Information et de la Communication. Direction de Viviane Couzinet. Toulouse: LERASS ; Université Paul Sabatier III, 2008.

KERR PINHEIRO, M. M. Processo de transformação das políticas de informação no estado informacional. Pesq. bras. ci. inf., Brasília, v. 3, n. 1, p. 113-126, jan./dez. 2010

KERR PINHEIRO, M. M. Política de informação no Estado informacional: um documento de interesse público. In: DEUXIÈME COLLOQUE SCIENTIFIQUE INTERNATIONAL DU RÉSEAU MUSSI, 10., 2011, Toulouse. Actes du Deuxième Colloque scientifique international Du Réseau MUSSI : médiations et hybridations. Toulouse: Universidade de Toulouse 3 IUT, 2011. p. 173-184.

LIMA C. R. A. et al. A experiência de disseminação de informações em Saúde. In: MINISTÉRIO DA SAÚDE. ORGANIZAÇÃOO PAN-AMERICANA DA SAÚDE. FUNDAÇÃO OSWALDO CRUZ. A experiência brasileira em sistemas de informação em saúde. Produção e disseminação de informações sobre saúde no Brasil. Brasília - DF: Ministério da Saúde, 2009. v. 1. p. 109128. (Série $B$. Textos Básicos de Saúde).

MARQUES, R. M.; KERR PINHEIRO, M. M. Política de informação nacional e assimetria de informação no setor de telecomunicações brasileiro. 
Perspectivas em Ciência da Informação, v. 16, n. 1, p. 65-91, jan./mar. 2011

MOORE. La soeté de I'informacion/UNESCO: rapport Mondial sur I'information 97/98. Paris: UNESCO, 1997. $3^{\text {ème }}$ partie, chap.20, p.289302.

MORAES, I. H. S. Política, tecnologia e informação em saúde: a utopia da emancipação. Salvador, BA: Casa da Qualidade Editora, 2002. 171p.

MORAES, I. H. S.; VASCONCELLOS, M. M. Política nacional de Informação, Informática e Comunicação em Saúde: um pacto a ser construído. Saúde em Debate, Rio de Janeiro, v. 29, n. 69, p. 86-98, jan./abr. 2005.

OLIVEIRA, M. M. Como fazer pesquisa qualitativa. Petrópolis: Vozes, 2007. 